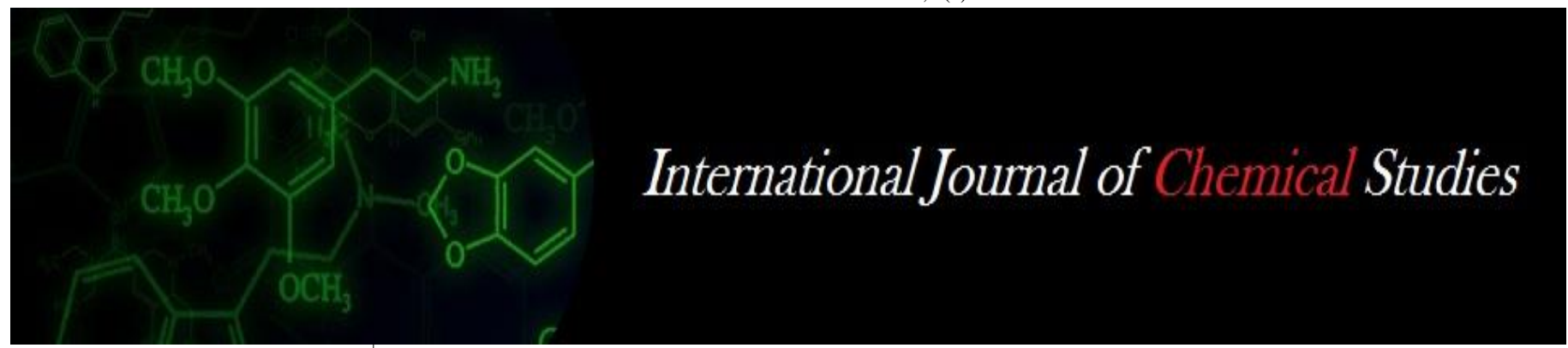

P-ISSN: 2349-8528

E-ISSN: 2321-4902

www.chemijournal.com

IJCS 2020; 8(2): 894-897

(C) 2020 IJCS

Received: 04-01-2020

Accepted: 06-02-2020

Priti Jain

Department of Post Harvest

Process and Food Engineering,

College of Agricultural

Engineering, JNKVV, Jabalpur,

Madhya Pradesh, India

Corresponding Author:

Priti Jain

Department of Post Harvest

Process and Food Engineering,

College of Agricultural

Engineering, JNKVV, Jabalpur,

Madhya Pradesh, India

\section{Effect of spray drying process parameters on particle morphology for fermented beetroot powder}

\section{Priti Jain}

DOI: $\underline{\text { https://doi.org/10.22271/chemi.2020.v8.i2n.8880 }}$

\section{Abstract}

This study covers the morphological changes during the production of spray-dried fermented beetroot juice powder to keep the product quality. Particle morphology was observed by different processes parameters such as inlet air temperature, airflow rate, feed flow rate, and maltodextrin concentration. Particle morphology was analyzed by Scanning Electron Microscopy (SEM). It was remarked from SEM images of the particle that agglomeration potential was directly proportional to airflow rate and feed flow rate whereas smooth surface and larger sizes of particles were perceived at higher inlet air temperature and maltodextrin concentration.

Keywords: Beetroot, fermentation, spray drying, morphology, scanning electron microscope

\section{Introduction}

Beetroot (Beta vulgaris L.) named 'chukander', is mainly cultivated in India for its juice and vegetable value. It is a biannual crop. It is the most powerful source of nutrients available for overall human health. Beetroot powder is reported to have medicinal properties, particularly to improve digestion and blood quality ${ }^{[1,2]}$.

Dehydration through spray drying is one technique widely used in the food industry, and under optimal processing conditions, it has been proven to be an effective method to obtain various products. During the drying process, the feed solution is sprayed in droplets in a stream of hot air ${ }^{[3]}$. The quality of the spray-dried powder is quite dependent on the spray-dryer operating parameters. These parameters include: drying agent material, feed flow rate, airflow rate, inlet, and outlet air temperature should be tested.

The shell structure of a drying droplet plays an important role in determining the morphology of the final dried particle ${ }^{[4]}$. The main characteristics of spray-dried powders are the particle size (granulometry) and particle shape (morphology). The morphology of powder particles indicates their physical structure. The physical structure of particle changes due to process parameters which affect the powder properties. Particle morphology and particle size distributions are two critical factors in determining properties of dried powder (wettability, stability, and cohesiveness or dispersibility) and their application in composite ${ }^{[5,6]}$.

Different electron microscopy techniques including transmission electron microscopy, scanning electron microscopy, cryo-SEM, cryo-TEM, and environmental scanning electron microscopy have been employed for the evaluations related to the process of food. Scanning electron microscopy is a very useful tool to visualize food structure because; it combines in many ways the best features of light microscopy and transmission electron microscopy. SEM process is mostly used for the analysis of particle structure. These include relatively simple sample preparation, a wide range of magnification, high depth of field and the fact that the image is a representation of electronic data allowing for image analysis and quantification.

However, the morphology study for spray-dried fermented beetroot powder has not been previously reported. Therefore, the main goal of the current work is to investigate the effects of spray drying process parameters on particle morphology for fermented beetroot powder.

\section{Materials and Methods}

Raw Materials and fermented juice preparation 
Freshly beetroot was procured from the local market, Jabalpur (M.P.) for this study. The beetroots were cleaned with water to remove all dirt adhering and the pesticide residues. The sorted beetroots were cut into small pieces and juices were extracted. Prepared juice was fermented with the culture (saccharomyces cerevisiae MTCC 178) of $10 \% \quad(\mathrm{v} / \mathrm{v})$ inoculum and it was incubated at $25{ }^{0} \mathrm{C}$ for $24 \mathrm{hr}$. Then maltodextrin was added in it with the help of magnetic stirrer.

The spray drying process for the development of powder The powder was produced using a lab spray dryer (Labultima LU-222 Advanced Model, Twin Cyclone) with a different combination of process parameters. During the drying process, the fermented beetroot juice was sprayed in droplets in a stream of hot air. The liquid droplets of juice were dried in seconds as a result of the highly efficient heat and mass transfers. Process parameters for the production of powder were taken as inlet air temperature $(140,142,144,146$ and $\left.148^{\circ} \mathrm{C}\right)$, airflow rate $\left(49.56,51.92,54.28,56.64\right.$ and $\left.59 \mathrm{~m}^{3} / \mathrm{h}\right)$, feed flow rate $(120,127.5,135,142.5$ and $150 \mathrm{ml} / \mathrm{h})$ and maltodextrin concentration $(4,5,6,7$ and $8 \%$ ). They were optimized by response surface methodology of design expert 8.0.7.1. The obtained powder was then collected and weighed for analysis.

\section{Electron microscopy of spray-dried fermented beetroot powder}

Scanning electron microscopy was used for morphology study of different samples which were prepared by the spray drying process. SEM images of dried samples were recorded using a variable pressure scanning electron microscope (LEO 435VP) at AIIMS New Delhi. The samples were atomized on a small barrel. Then they were covered with a fine layer of gold through sputter coating attachment of Balzers, in vacuumed evaporators. For observation, a Scanning Electronic Microscope (Levi Zeiss Machine) working with a voltage of $15 \mathrm{kV}$ was used. The microphotographs were carried out with a camera coupled to the microscope. The samples were systematically observed with 750 and 1500x of magnification (Fig 1).

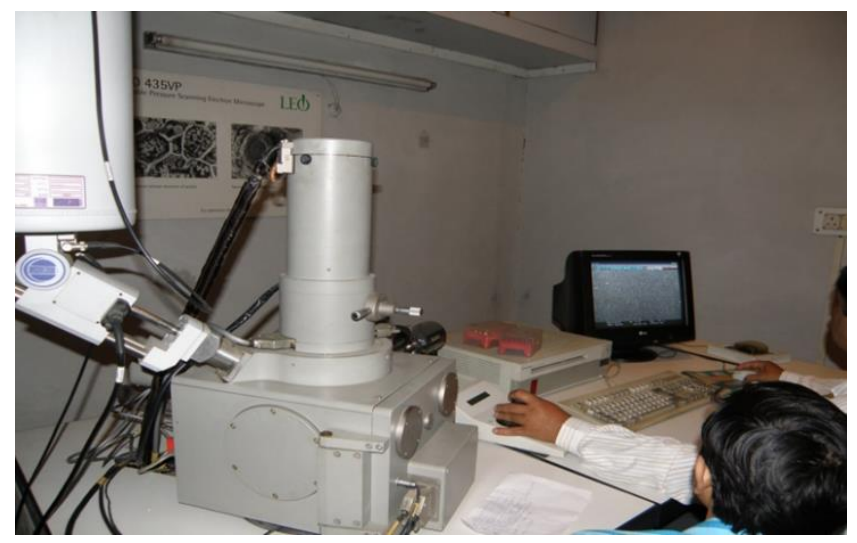

Fig 1: Particle morphology by Scanning Electron Microscopy

\section{Results and Discussion}

The morphology of spray-dried fermented beetroot powder is an essential parameter because it determines the physical construction of the particle. In this process, particles were affected by different process parameters such as inlet air temperature, airflow rate, feed flow rate, and maltodextrin concentration. Obtained particles from different drying conditions were found in different shape, size and agglomeration potential. Scanning electron microscopy images of spray-dried fermented beetroot powder obtained at different process conditions were studied as the following explanation.

\section{Effect of inlet air temperature}

The effects of inlet air temperature on particle morphology were showed in (Fig 2). An increase in inlet air temperature from 140 to $148{ }^{\circ} \mathrm{C}$ resulted in a greater number of particles with a smooth surface and larger sizes, i.e. particles were produced at high temperature with a smoother surface. This may be due to rapid water evaporation means higher drying rates and higher pressure inside the particles during microencapsulation preventing shrinking. At low temperature, Water diffusion allows more time for the particles to deform, wrinkle, and collapse. Similar results were reported by Beirao-da-Costa et al., Medina-Torres et al., and Tonon et al., [7, 9]. Some of the larger particles show some "deflating", due to softness of the shell during drying, which collapses as moisture is lost from the inside of the particle (Fig 3 ).

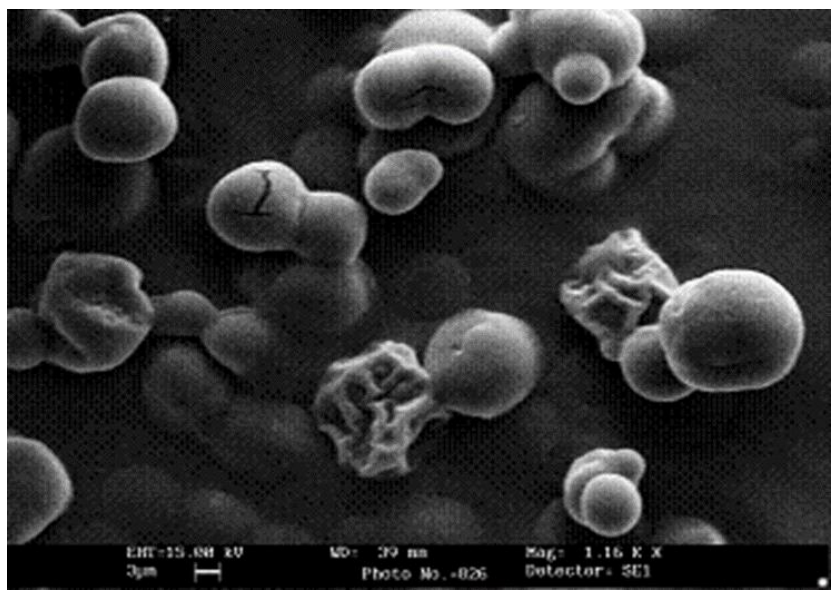

Fig 2: Microphotographs of particles obtained at inlet air temperature $\left(148{ }^{\circ} \mathrm{C}\right)$ and constant other parameters: air flow rate of $57 \mathrm{~m}^{3} / \mathrm{h}$, feed flow rate of $120 \mathrm{ml} / \mathrm{h}$ and maltodextrin concentration of $6 \%$

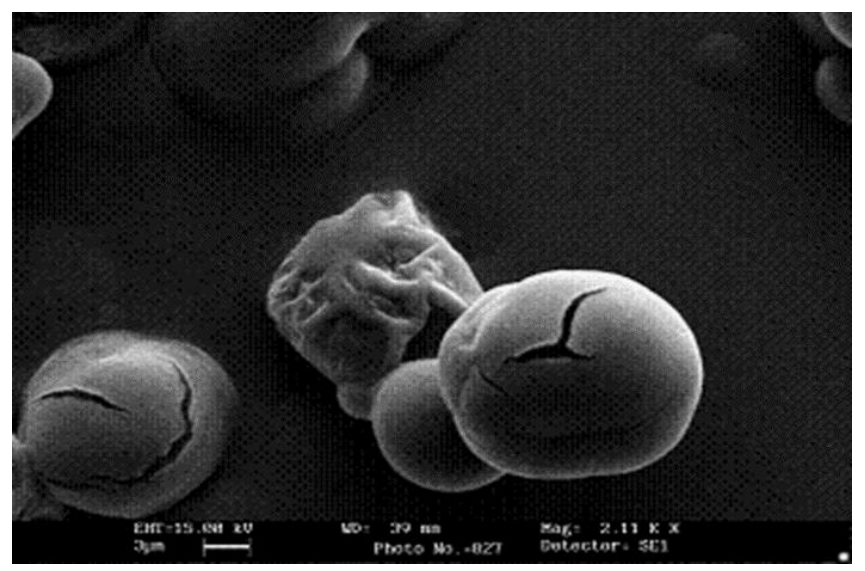

Fig 3: Microphotographs of particles obtained at higher inlet air temperature $\left(148^{\circ} \mathrm{C}\right)$ and constant other parameters

\section{Effect of airflow rate}

The increase in airflow rate from 49.56 to $59 \mathrm{~m}^{3} / \mathrm{h}$ increased agglomeration potential for any other constant parameters such as inlet air temperature, feed flow rate and maltodextrin concentration. Higher aspirator speed resulted in reduced residence time in the drying chamber and the consequently larger amount of residual moisture in the end product. Microphotographs (Fig 4) of beetroot powder obtained at 
lower air flow rate show a spherical and varied in size with a high proportion of agglomerates.

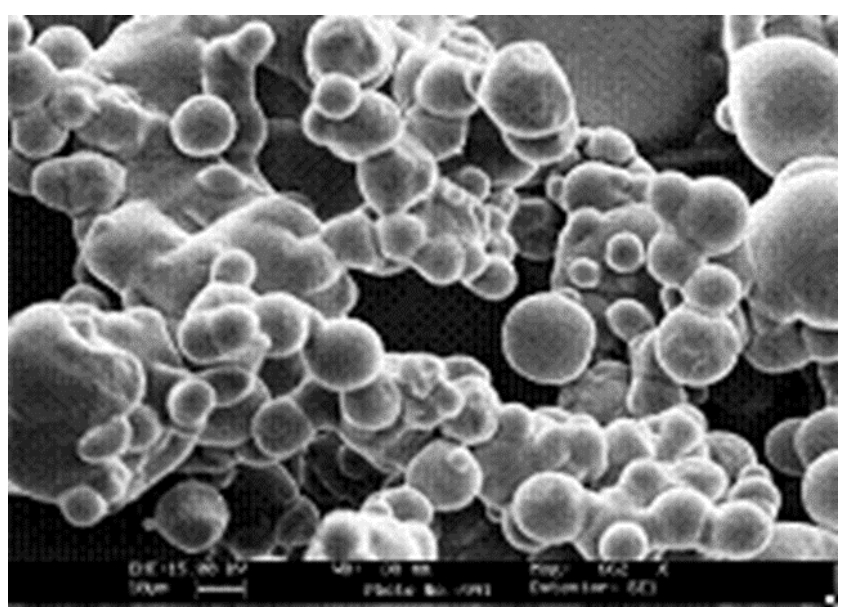

Fig 4: Microphotographs of particles obtained at lower air flow rate $\left(49 \mathrm{~m}^{3} / \mathrm{h}\right)$ and constant other parameters: inlet air temperature of 144 ${ }^{\circ} \mathrm{C}$, feed flow rate of $120 \mathrm{ml} / \mathrm{h}$ and maltodextrin concentration of $6 \%$

\section{Effect of feed flow rate}

Agglomeration potential was higher with an increase in feed flow rate from 120 to $150 \mathrm{ml} / \mathrm{h}$. The possible explanation for an increase in agglomeration potential may be because the capacity of the air volume was not sufficient to reduce the moisture content as high as possible, resulting in high agglomeration potential of the end product. Agglomeration occurs due to retained moisture in a particle as a result of adhesion forces between particles. In (Fig 5), clusters were seen which are collapsed. Depression ducts are there due to shrinkage. Thick Concentrated Clusters are visible due to more moisture, rock type clusters, variation in size is definite.

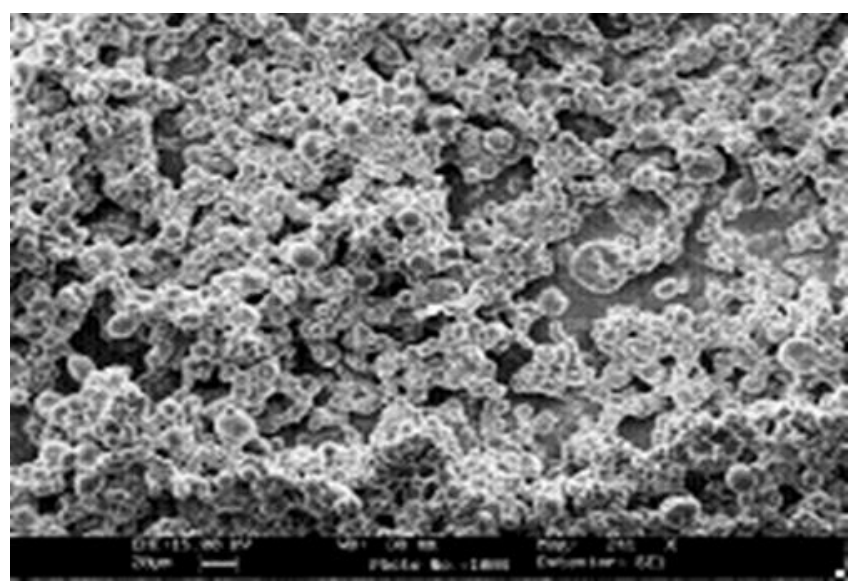

Fig 5: Microphotographs of particles obtained at higher feed flow rate $(150 \mathrm{ml} / \mathrm{h})$ and constant other parameters: inlet air temperature of $144{ }^{\circ} \mathrm{C}$, air flow rate of $57 \mathrm{~m}^{3} / \mathrm{h}$ and maltodextrin concentration of $6 \%$

\section{Effect of maltodextrin concentrations}

Larger particles were found with an increase in a maltodextrin concentration from 4 to $8 \%$ to the fermented beetroot juice before spray drying. Image of spray-dried fermented beetroot juice powder obtained at maltodextrin concentration of $8 \%$ is shown in (Figure 6). It is verified that the concentration of maltodextrin increased, the particles became more spherical and more scattered. As explained by Ferrari et al., ${ }^{[10]}$ also, particles produced with lower maltodextrin concentrations were smaller.

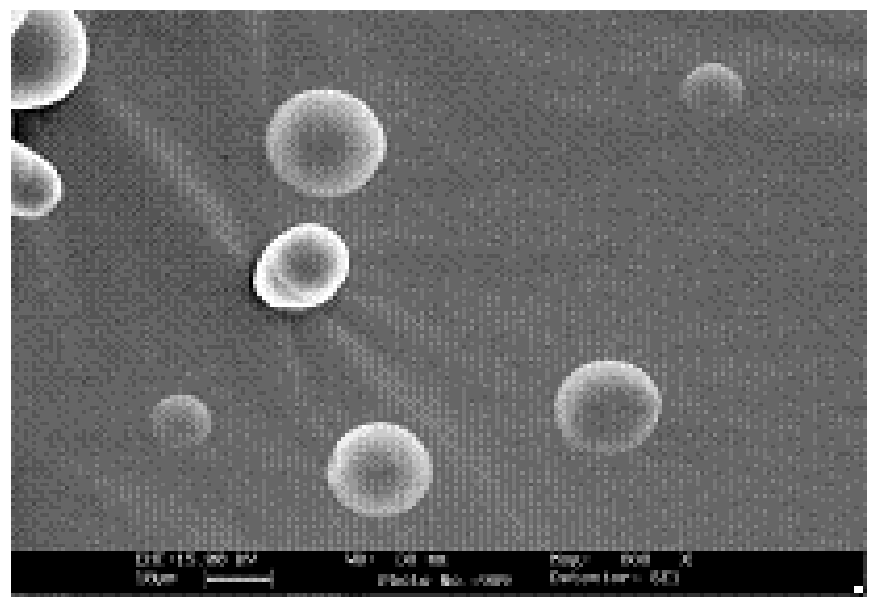

Fig 6: Microphotographs of particles obtained at higher maltodextrin concentration (8\%) and constant other parameters: inlet air temperature of $144^{\circ} \mathrm{C}$, air flow rate of $57 \mathrm{~m}^{3} / \mathrm{h}$ and feed flow rate of $120 \mathrm{ml} / \mathrm{h}$

\section{Conclusion}

The present study was concluded that the particle morphologies for fermented spray-dried beetroot powder were affected with different process parameters. Agglomeration potential was directly proportional to the airflow rate and feed flow rate. Particles were perceived at higher inlet air temperature and maltodextrin concentration with a smooth surface and larger sizes. The spherical shape of particles varied in size with a high proportion of agglomerates and visible cracks on surface predominant fermented spray-dried beetroot powder. With an increase in the concentration of maltodextrin the particles tended to become more spherical, more scattered. The SEM photographs indicated the most desirable microstructure of powder in fermented beetroot juice powder.

\section{Acknowledgement}

The authors are grateful to the Jawaharlal Nehru Krishi Vishwa Vidyalaya, Jabalpur, M.P., India for financial grant. We are also grateful to the Department of Post Harvest Process and Food Engineering for their support in carrying out a part of the reported research work. We thank the Department of Food Science for providing lab and instrumental facilities.

Conflict of interest Statement: The authors declare that there is no conflict of interest.

\section{References}

1. Delgado- Vargas F, Jiménez AR, Paredes-López O, Natural pigments: carotenoids, anthocyanins, and betalains--characteristics, biosynthesis, processing, and stability. Food Sci Nutr. 2000; 40(3):173-289.

2. Stintzing FC, Carle R. Functional properties of anthocyanins and betalains in plants, food, and in human nutrition. Trends Food Sci Technol. 2004; 15(1):19-38.

3. Saravacos GD, Kostaropoulos AE. Handbook of Food Processing Equipment. Kluwer Academic/Plenum Publishers, New York, 2002.

4. Lin JC, Gentry JW. Spray drying drop morphology: Experimental study, Aerosol Sci Technol. 2003; 37(1):15-32.

5. Mocoz J, Pukenszky B. Polymer micro and nanocomposites: Structure, interactions, properties. J Ind Eng. Chem. 2008; 14:535-563. 
6. Vehring R. Pharmaceutical particle engineering via spray drying. Pharm Res. 2007; 25(5):999-1022.

7. Beirao-da-Costa S, Duarte C, Bourbon AI, Pinheiro AC, MIN, Vicente AA, Beirão-da-Costa ML, Delgadilo I, Inulin potential for encapsulation and controlled delivery of Oregano essential oil. Food Hydrocoll. 2013; 33(2):199-206.

8. Medina-Torres L, Santiago-Adame R, Calderas F, Gallegos-Infante JA, González-Laredo RF, RochaGuzmán NE, Núñez-Ramírez DM, Bernad-Bernad MJ, Manero O. Microencapsulation by spray drying of laurel infusions (Litsea glaucescens) with maltodextrin,. Ind. Crop Prod. 2016; 90:1-8.

9. Tonon, R. V., Brabet, C. and Hubinger, M. D., Influence of process conditions on the physicochemical properties of acai powder produced by spray drying, J Food Eng., 2008, 88, 411-418.

10. Ferrari CC, Germer SPM, De Aguirre JM. Effects of spray-drying conditions on the physicochemical properties of blackberry powder, Dry Technol. 2012; 30:154-163. 